\title{
Estimating age-dependent per-encounter chlamydia trachomatis acquisition risk via a Markov-based state-transition model
}

\author{
Yu Teng ${ }^{1}$, Nan Kong ${ }^{1 *}$ and Wanzhu Tu ${ }^{2}$
}

\begin{abstract}
Background: Chlamydial infection is a common bacterial sexually transmitted infection worldwide, caused by $C$. trachomatis. The screening for $C$. trachomatis has been proven to be successful. However, such success is not fully realized through tailoring the recommended screening strategies for different age groups. This is partly due to the knowledge gap in understanding how the infection is correlated with age. In this paper, we estimate age-dependent risks of acquiring C. trachomatis by adolescent women via unprotected heterosexual acts.

Methods: We develop a time-varying Markov state-transition model and compute the incidences of chlamydial infection at discrete age points by simulating the state-transition model with candidate per-encounter acquisition risks and sampled numbers of unit-time unprotected coital events at different age points. We solve an optimization problem to identify the age-dependent estimates that offer the closest matches to the observed infection incidences. We also investigate the impact of antimicrobial treatment effectiveness on the parameter estimates and the differences between the acquisition risks for the first-time infections and repeated infections.
\end{abstract}

Results: Our case study supports the beliefs that age is an inverse predictor of C. trachomatis transmission and that protective immunity developed after initial infection is only partial.

Conclusions: Our modeling method offers a flexible and expandable platform for investigating STI transmission.

Keywords: Chlamydial infection, Acquisition risk, Transmission probability, Parameter estimation, State transition model

\section{Background}

Chlamydial infection, caused by the bacterium, C. trachomatis, is a commonly reported sexually transmitted infection (STI) worldwide [1]. It can be accurately diagnosed and effectively cured if being treated promptly [2-4]. On the other hand, the infection may go unnoticed for many years and consequently lead to severe morbidities, including pelvic inflammatory disease, ectopic pregnancy, tubal pregnancy, preterm birth, and increased susceptibility of HIV infection [5-13]. Therefore, it is important to the high-risk individuals and social groups as well as societies in general to schedule screening tests at the right time.

The above clinical facts promote the model-based analysis of screening programs for chlamydial infections

\footnotetext{
* Correspondence: nkong@purdue.edu

'Weldon School of Biomedical Engineering, Purdue University, 206 S. Martin Jischke Dr, West Lafayette, IN 47907, USA

Full list of author information is available at the end of the article
}

[14-19]. Based on the evidence of high C. trachomatis prevalence among adolescents and remaining risk for repeated infection among those who were recently treated for infection [20-27], routine population screening for female adolescents, especially those who were recently infected, has been suggested to be cost-effective, and in some cases, cost-saving [18,28-31]. At present, some routine screening strategies are endorsed by clinical practice guidelines [32-34] and recommended to adolescents during their health visits $[5,35,36]$. However, when tailoring such strategies (e.g., specifying the screening frequency) with respect to age and prior infection status, we face the challenge of lacking reliable epidemiological data. Understanding the acquisition risk differences with respect to age and prior infection status may offer insights into the mechanism of C. trachomatis acquisition and chlamydial infection. Such understanding will lead to more detailed model-based economic studies on the 
effectiveness and cost-effectiveness of screening strategies, which has the potential to further improve the prevention of chlamydial infection.

In this paper, we use observational infection data to estimate age-dependent per-encounter $C$. trachomatis acquisition risks, i.e., the probability that a female subject is infected with C. trachomatis through an unprotected coital event. For brevity, we use acquisition risk for referring to per-encounter C. trachomatis acquisition risk. It is unethical to design controlled experiments that expose human subjects to infectious pathogens. This challenge is alleviated with observational studies and model-based studies. Katz [37] and Tu et al. [38] used cross-sectional data and longitudinal data to estimate the acquisition risk, respectively. The estimation was also described in several model-based studies for screening program/strategy evaluation. Kretzschmar et al. [14] developed an individualbased stochastic simulation model to describe the spread of C. trachomatis in a heterosexual population with a highly sexually active core group. The authors used Monte-Carlo simulation to estimate the daily transmission rates. The data source used in this paper for sexual behavior and partnerships were based on a survey conducted in the Netherlands in 1989 [39]. Kretzschmar et al. [15] used the simulation model in [14], acquired the per partnership transmission probability from [40], and applied the method in [37] to estimate the per-act transmission probability. Turner [17] used a more comprehensive stochastic network model based on Ghani et al. [41] for the estimation. The network includes not only disease transmission and recovery but also dynamic partnership choice, formation, and dissolution. The transmission probabilities per sex act were estimated by systematic fitting to a variety of appropriate UK-based data sources $[42,43]$. Other studies on the transmissibility of $C$. trachomatis include Gray et al. [44], Lycke et al. [45], Ruijs et al. [46], and Vickerman et al. [47]. However, none of the papers above investigated the age- and prior-infection-dependency on C. trachomatis transmissibility. Additionally, many model-based studies used multiple data sources and relied on expert opinion driven model assumptions. For a list of works on estimating or using C. trachomatis transmission probabilities, we refer to Additional file 1.

To estimate age-dependent acquisition risks, we developed a Markov-based individual state-transition model that describes the changes in states of infection for each subject over time. The transition probabilities in the model are time-varying. In addition, we extended our model to investigate the difference on the acquisition risk that leads to a first-time infection and leads to a repeated infection. Furthermore, we varied the effectiveness of antimicrobial treatment to assess its impact on the acquisition risk estimates. Methodologically, the approach proposed is a novel application of time-varying Markov modeling with longitudinally observed infection data. It allows us to assess the probability of failures in antimicrobial treatment. To our knowledge, only Tu et al. [38] applied a similar approach of using longitudinal data to estimate the transmissibility of C. trachomatis. However, they did not consider the potential age-dependency in the changes on the infection state nor differentiate first-time infection and repeated infection. While there are many articles estimating the transmissibility of HIV/AIDS (e.g. [48,49]), few statistical approaches (e.g. [38]) were developed for bacterial STI with additional data and methodological challenges. Unlike HIV/AIDS, infections with STI bacteria are routinely treated and effectively cured. This causes a shift in the state of infection and poses greater methodological challenges on quantifying the transmissibility. Meanwhile, frequently measured infection data are needed to capture such infection dynamics. To our knowledge, few studies have been designed to collect such data. In the next section, we describe an observational study providing ideal data for our research.

The acquisition risk is a population-specific quantity, which reflects not only the transmissibility of the $C$. trachomatis organism but also the organism's prevalence in the male partner population. Clearly, higher acquisition risks are associated with the organism being more transmissible and being more prevalent among male partners. Given $C$. trachomatis prevalence in the male partner population, one can quantify the male-to-female $C$. trachomatis transmission probability for young women within a particular age group to be the ratio between the per-encounter $C$. trachomatis acquisition risk within the particular age group and the prevalence among the male partners that are associated with the young women of that age group, i.e., per-encounter C. trachomatis acquisition risk $=$ transmission probability $\times$ prevalence in the male partner population. For our case study, we used the observational data on recurrent sexually transmitted disease among the recruited adolescent women. Because the exact prevalence of $C$. trachomatis infection in the male partner population was not attained in the study used, we in this paper focus on the estimation of acquisition risks, which reflects the transmission risk presented in the male partner population.

The remainder of the paper is organized as follows. In Section 2, we describe the observational data set used in the parameter estimation. In Section 3, we describe our estimation method. In Section 4, we report our estimates based on the data set and discuss the results. We draw conclusions, discuss limitations, and outline future research in Section 5.

\section{Description of the analyzed data set}

The observational data used in our case study were collected through the "Young Women's Project" (YWP), 
which is an epidemiological study of recurrent STI in adolescent women recruited from an inner city population that was at increased risk of STI. The YWP started its enrollment in 1999. Its study design and data collection provide a platform for the estimation of the $C$. trachomatis transmissibility. We present the data collection scheme in Figure 1.

In a nutshell, young women between the ages of 14 and 17 years old were identified and recruited by the project. The recruitment criteria used included 1) understanding English; 2) no serious mental or psychiatric health problems; and 3) visiting one of three participating primary care clinics. For those young women who met all the recruitment criteria, they would be approached by research staff and asked for enrollment. Note that these criteria do not depend on subjects' prior sexual experience, which ensures the randomness of the cohort. To enroll an YWP study subject, she received initial interviews and underwent a pelvic examination, during which a cervical swab for STI testing was collected and analyzed with nucleic acid amplification test for $C$. trachomatis. Infected participants were treated while at the clinics or shortly after the test results became available. Enrolled participants were then asked to visit their clinics on a quarterly basis. At each follow-up visit during the study period, enrolled participants underwent STI testing and treatment. Also at each follow-up visit, the participants received follow-up interviews, in which they were queried about the number of unprotected coital events since previous visit. Most of the participations did not visit the clinic every quarter and left the project before its completion.

For our analysis, we collected 1173 quarterly test results from the first 200 participants who were never infected and have completed at least two follow-up visits. Their average enrollment age was 15 years with standard deviation of 1.1 years. These participants underwent averagely 5.86 visits, ranging from 2 to 18 . They stayed in the project for 8.2 years maximally and 3.2 years on average. They reported averagely 14.3 quarterly unprotected coital events. C. trachomatis was detected from 208 of the quarterly swab samples, equivalent to $17 \%$. For more information on the YWP data collection and the observational data set analyzed for this paper, we refer to Tu et al. [38].

\section{Description of the estimation method}

For the estimation, we first developed an age-dependent Markov state-transition model that depicts the disease condition dynamics for each female individual (Figure 2). In the model, we let $C$ and $I$ be the states where a female subject does not and does have $C$. trachomatis, respectively. Without loss of generality, we assume that the age range we study is $\left[T_{1}, T_{2}\right]$. We also assume $\Delta t$ to be the unit-time interval during which no state transition occurs and all transition rates remain the same. With the YWP, the smallest length for $\Delta t$ is set to be a quarter year, the time interval between two consecutive visits. Given the studied age range $\left[T_{1}, T_{2}\right]$ and the unit-time interval $\Delta t$, we index the discrete age points to be $\left.\mathrm{i}=0,1, \Gamma\left(T_{2}-T_{1}\right) / \Delta t\right\rceil$ with 0 indexing $T_{1}$ and $N \equiv\left\ulcorner\left(T_{2}-T_{1}\right) / \Delta t\right\rceil$ indexing $T_{2}$. We denote $p_{i}$ to be a constant acquisition risk between discrete age points $i$ and $i+1$ for $i=0,1, \ldots, N-1$. That is, when a subject of age point $i$ is at state $C$, we assume that the subject follows a constant probability to transition to state $I$ with unprotected coital events between the two consecutive age points. Once a subject is at state $I$ at age point $i$, the only reason she does not transition back to state $C$ at the next discrete time point is due to the ineffectiveness of the antimicrobial treatment. We use $q$ to measure the treatment effectiveness with the assumption that this quantity is constant irrespective of the age. That is, $q$ is the probability that a subject transitions to state $C$ given she is currently at state $I$. Lastly, we denote $t_{\mathrm{en}} \geq T_{1}$ and $t_{\mathrm{ex}} \leq T_{2}$ to be the entry and exit ages of a female subject, respectively. We can conveniently map

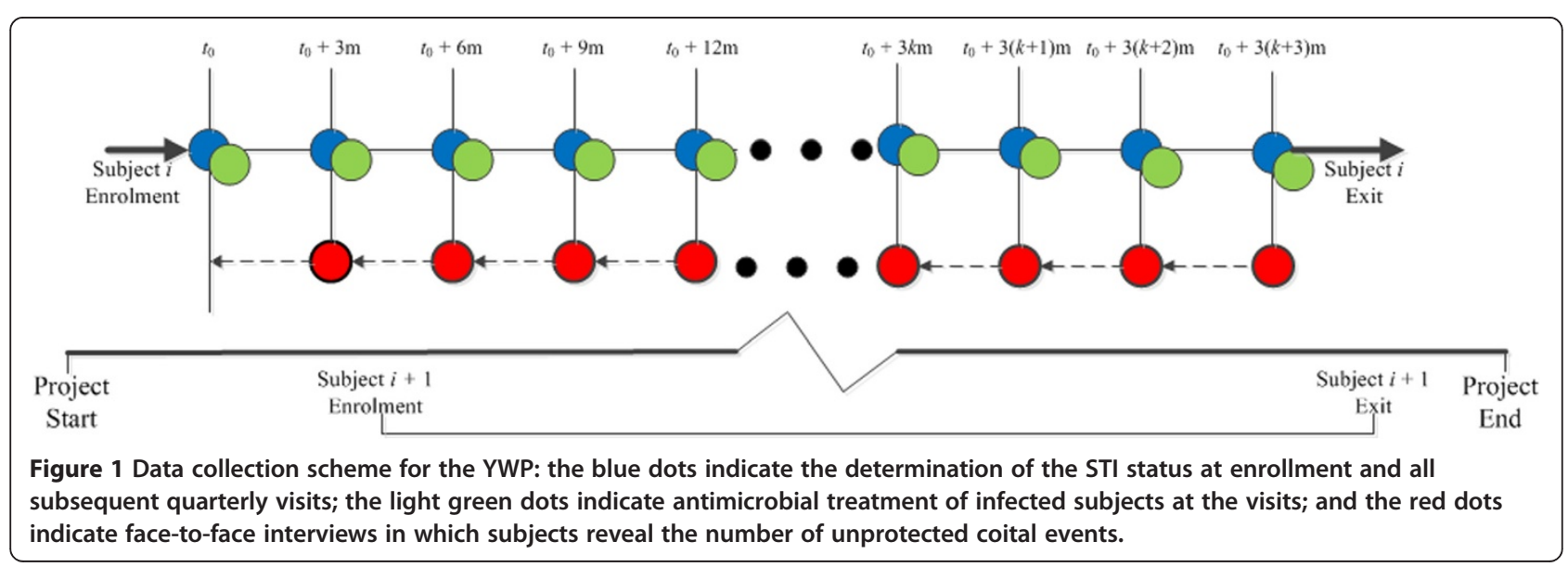




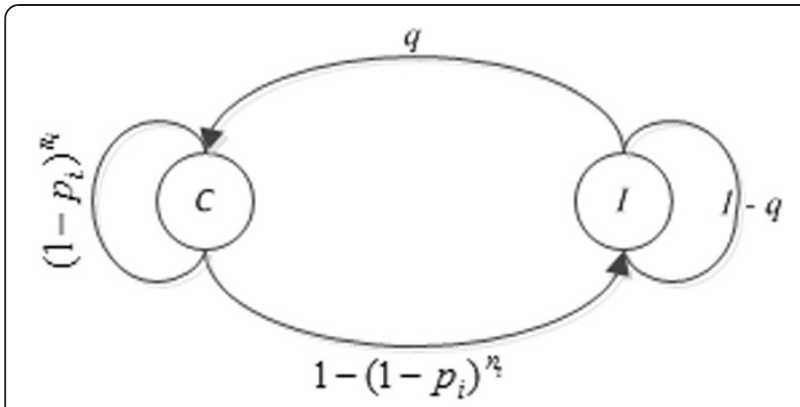

Figure 2 State transition diagram of the age-dependent Markov model that captures the disease condition dynamics for chlamydial infection and curing at discrete age point $i=0,1, \ldots$, $N-1$.

the two age values to two age point indices between 0 to $N$. We call them $i_{a} \geq 0$ and $i_{b} \leq N$.

Let $s_{i}$ be the state of a female subject between age point $i$ and $i+1$ with $i_{a} \leq i<i_{b}$. Let $n_{i}$ be the number of unprotected coital events with an infected partner during the same period. Assuming that the probability that a female subject acquires C. trachomatis is independent between any two unprotected coital events, the transition probabilities are presented as: $\operatorname{Pr}\left(s_{i+1}=C \mid s_{i}=C\right)=$ $\left(1-p_{i}\right)^{n_{i}}$, which means that to ensure no infection, the subject does not acquire $C$. trachomatis in any of the unprotected coital events during the period. Here we also assume a female subject has zero probability of acquiring C. trachomatis from a protected coital event. Note that a similar idea can be found in Katz [37]. It is easy to determine the other transition probabilities in the statetransition model.

We next developed a cubic spline model, based on the longitudinal study in $\mathrm{Tu}$ et al. [38], to predict the number of unprotected coital events with an infected partner during the next quarter year at age $t$ (in years). The model is presented as:

$$
n(t)=\exp \left\{\beta_{0}+\beta_{1} t+\beta_{2} t^{2}+\beta_{3} t^{3}+\sum_{i=1}^{N} u_{i}\left(\left(t-k_{i}\right)_{+}\right)^{3}\right\} .
$$

A cubic spline model is a polynomial function that is piecewise-defined and possesses smoothness of order 3 . For each discrete age point $i=0,1, . ., N-1$, we can use its corresponding age, denoted by $t_{i}$, to calculate $n\left(t_{i}\right)$ and specify $n_{i}$. The expression $(a)_{+}$indicates the value of $a$ is kept when it is nonnegative and its value is set to be 0 when negative. Given the data availability, the model shows good fit for the age range between years of 15 and 24.75. In the model, $N$ indicates the number of knots and is set to be 40 . The parameters $k_{i}$, termed knots, indicate age points between 14.5 and 24.5. These knots are the places in the model where the polynomial pieces connect. The parameters $u_{i}$, together with the base function $\beta_{0}+\beta_{1} t+\beta_{2} t^{2}+\beta_{3} t^{3}$, ensure that the estimates of the spline model match the corresponding observations exactly at those age points. We report the model parameter values in Additional file 2.

As an extension to incorporate cohort variation on the number of unprotected coital events, we assume the intercept (i.e., the first term $\beta_{0}$ ) of the cubic spline model for each simulated subject to be normally distributed with mean being the intercept from the original model and standard deviation being a percentage of the mean. Once the intercept was sampled for each simulated subject, we adjusted the corresponding cubic spline model but kept the values of the knots the same for all the subjects to ensure the necessary correlation among different time points. Thus the extended stochastic model is presented as:

$$
n(t)=\exp \left\{u_{0}+\beta_{0}+\beta_{1} t+\beta_{2} t^{2}+\beta_{3} t^{3}+\sum_{i=1}^{N} u_{i}\left(\left(t-k_{i}\right)_{+}\right)^{3}\right\},
$$

where $u_{0}$ follows a normal distribution as $u_{0} \sim N\left(0,\left(\rho \times \beta_{0}\right)^{2}\right)$ with $0<\rho \leq 1$.

To estimate age-dependent acquisition risks, we applied a reverse engineering approach with real age-specific chlamydial infection incidences extracted from the observational data. For any candidate age-dependent acquisition risk profile, we simulated the infection incidence given a collection of hypothetical female subjects, each of which is assigned a random enrollment age and a random exit age, based on the same observational data. For each subject, we also followed the stochastic model above to uniquely determine for each hypothetical subject the number of unprotected coital events in all discrete age points from the simulated enrollment to the simulated exit. With the simulation of each subject, we recorded the age points within which each infection occurred to the subject. Finally, we tallied the number of chlamydial infections for the entire simulated cohort within each age group.

We constructed an optimization problem to compute the acquisition risks with which the simulated incidences match the observed ones most closely. To present the optimization problem, let us introduce additional mathematical notation. We use $\bar{I}_{i}$ to denote the observed percentage of infected in the studied cohort for age group $i=1, \ldots$, $N$. We use $K$ to denote the set that contains the indices of the simulation runs. For each age group $i$, we collect all hypothetical subjects that experienced $i$ during their enrollment (i.e., the age corresponding to $i$ is between the subject's entry and exit ages). We denote $K(i) \subseteq K$ to be the subset that contains the simulation run indices for such subjects. For each simulation run $k \in K(i)$, we use $I_{i}(k) \in$ $\{0,1\}$ to indicate whether an infection occurs for age 
group $i=1, \ldots, N$. The objective of the optimization problem is to minimize the difference between $\frac{1}{|K(i)|} \sum_{k \in K(i)} I_{i}(k)$, the simulated percentage of infected, and $\bar{I}_{i}$ for each $i=1, \ldots, N$. The optimal solution specifies the age-dependent acquisition risk, which is denoted by $\bar{p}=\left(\bar{p}_{1}, \ldots, \bar{p}_{N}\right)$.

We next considered the case where we distinguish the acquisition risks for first-time infection and repeated infection. This distinction is supported by the existing literature. Many studies, mostly involving women, have evaluated the risk of repeated infections during a period of observation and found that repeated infections are common during the first half a year after initially treated infections [21,22]. Some of these studies [25,26] also observed higher risk of repeated infections among younger women. We therefore extended the above Markov statetransition model (Figure 3). We let $C_{1}$ and $C_{2}$ be the states where a female subject is cured from chlamydial infection for the first time and reinfection, respectively, due to unprotected coital events. Similar to the first model, we assumed that both risks are independent between any two unprotected coital events. We denote $p_{t}^{1}$ and $p_{t}^{2}$ to be the two corresponding acquisition risks, and denote $n_{t}^{1}$ and $n_{t}^{2}$ to be the numbers of unprotected coital events before the first infection and after, respectively. Then we have $\operatorname{Pr}\left(s_{i+1}=I \mid s_{i}=C_{1}\right)=1-\left(1-p_{t}^{1}\right)^{n_{t}^{1}}$ and $\operatorname{Pr}\left(s_{i+1}=I \mid\right.$ $\left.s_{i}=C_{2}\right)=1-\left(1-p_{t}^{1}\right)^{n_{t}^{1}} 1-\left(1-p_{t}^{2}\right)^{n_{t}^{2}}$. Once a subject has been infected, she will only transition between $C_{2}$ and $I$, and never transition back to the state $C_{1}$.

To estimate age-dependent acquisition risks, we again applied a reverse engineering approach. We constructed two optimization problems to compute the acquisition risks that lead to first-time infection and reinfection. With the acquisition risks, the simulated incidences (i.e., first-time infection incidence and reinfection incidence) should match the observed ones most closely. To present the optimization problems, let us introduce additional

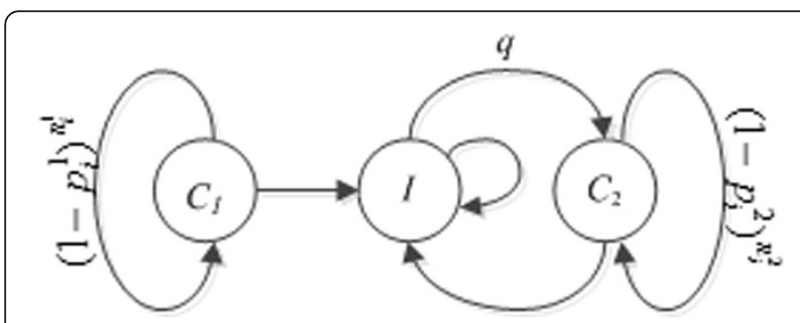

Figure 3 State transition diagram of the time-varying Markov model that captures the disease dynamics with first-time chlamydial infection, curing, and repeated infection. Note that the risks of chlamydial infection for the first time and repeated infection are different; and the sexual behaviors before and after the first-time infection are different as well. mathematical notation. We use $\bar{S}_{i}^{f}$ and $\bar{S}_{j}^{r}$, respectively, to denote the observed percentages of first-time infection and repeated infection in the studied cohort for age group $i, j=1, \ldots, N$. Similar to the above definition of $K(i)$, we introduce $K^{f}(i)$ and $K^{r}(i)$. For each simulation run $k$, we use $S_{i}^{f}(k)$ and $S_{j}^{r}(k)$, respectively, to indicate whether a first-time infection and a repeated infection occurs within each age group. The objectives of the two optimization problems are to minimize $\frac{1}{\left|K^{f}(i)\right|} \sum_{k \in K^{f}(i)} S_{i}^{f}(k)-\bar{S}_{i}^{f}$ and $\frac{1}{\left|K^{r}(i)\right|} \sum_{k \in K^{r}(i)} S_{i}^{r}(i)-\bar{S}_{i}^{r}$ for each $i=1, \ldots, N^{f}$ and $j=1, \ldots, N^{r}$, respectively. The optimal solutions specify the agedependent risks of acquiring C. trachomatis for the first time and reacquiring the bacterium, which are denoted by $\bar{p}^{f}=\left(\bar{p}_{1}^{f}, \ldots, \bar{p}_{N^{f}}^{f}\right)$ and $\bar{p}^{r}=\left(\bar{p}_{1}^{r}, \ldots, \bar{p}_{N^{r}}^{r}\right)$, respectively.

\section{Results}

In our numerical study, we used observational data collected through the YWP on age-specific chlamydial infection rate for the first time since the previous visit, denoted by $\mathrm{CT}$ rate (i), and age-specific cumulative $\mathrm{CT}$ infection rate (including both first-time infection and repeated infection), denoted by cum_CT_rate(i), for each age point $i$. We also tallied the total number of visits made by subjects at each age group, denoted by total_popu(i). For the first Markov model, we have $\bar{I}_{i}=C T$ rate $(i) \times$ total_popu $(i)$. We considered the age range from 15 to 24.75 with 3 months as the length of the fixed unit-time interval. That is, $N=40$. For the second Markov model, we have $\bar{S}_{i}^{f}=$ $(1$ - cum_CT_rate $(i)) \times$ total_popu $(i)$ and $\bar{S}_{i}^{r}=$ $($ cum_CT_rate $(i)-C T$ rate $(i)) \times$ total_popu $(i)$. Due to

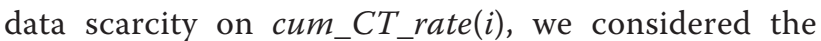
age range from 17 to 22 with 6 months as the length of the fixed unit-time interval for the second model. That is, $N=10$. For both Markov models, we used the cubic spline model described earlier to predict the number of unprotected coital events within each 3-month interval between age 15 and 25 . We introduced randomness to the cubic spline model by assuming a normal distribution on the intercept (i.e., the first term $\beta_{0}$ ) of the model and setting its standard deviation to be $10 \%$ of the intercept based on expert opinion, i.e., $\rho=10 \%$. Then we drew a sample from the normal distribution in each simulation run. In both models, we also varied $q$, the antimicrobial treatment failure probability, to be $0 \%$, $5 \%$, or $10 \%$. It is worth noting that the treatment failure probability may have significant variation among individual patients and clinics. That motivated us to conduct a sensitivity analysis. Lau and Qureshi [50] reported in a meta-analysis of randomized clinical trials that the failure probabilities of using azithromycin and 
doxycycline, the antibiotic considered in the YWP, to treat genital chlamydial infections were on average $3 \%$ and $2 \%$, respectively. The treatment failure probability in a realworld setting is less defined and difficult to measure. A recent article (Tu et al. [38]) suggested using $10 \%$ as the expected chance of a treatment failure. These numbers helped us specify the probability range for our sensitivity analysis.

We ran the simulation 10,000 times with generation of 10,000 hypothetical subjects for the study of each case described above. We report in Tables 1 and 2 the means at selected age points. From Table 1, we observed that 1) $\bar{p}$ monotonically decreases as age increases; 2$) \bar{p}$ decreases as $q$ increases. The first observation supports the evidence that the rate and prevalence of chlamydial infection are shown inversely related with age [51,52]. The second observation matches the intuition behind the relationship between treatment effectiveness and infection risk. If the treatment is less effective, the infected individual stays longer in state $I$ and thus takes a longer period of time to be infected again. This leads to a lower acquisition risk to match the observational data. From Table 2, we observed 1) $\bar{p}^{r}$ is positive; 2) both $\bar{p}^{f}$ and $\bar{p}^{r}$ monotonically decrease as age increases, and 3) $\bar{p}^{r}$ decreases as $q$ increases. The first observation support the concept that some degree of protective immunity against reinfection develops after first-time infection, although it appears to be partial at best $[53,54]$. The second observation supports the concept that acquired protective immunity may restrict chlamydia replication in older persons [55]. In other words, in addition to likely inverse relationship between age and unprotected sexual activity, organism load has been shown inversely related with age as well. As a result, many naive persons, considering themselves treated for initial infections successfully, may resume sexual activity at a level as active as before if not more active [22]. The third observation can be interpreted in the same way as earlier. Note that first-time infection occurs prior to any treatment. Hence, the estimate for the first-time infection is independent of the treatment effectiveness.

We also computed the standard deviations of the estimates over multiple simulation runs for both models. All the standard deviations on the estimates over multiple simulation runs are small relative to the mean estimates. This implies that the cohort variation on the risk estimates is nearly negligible when using $10 \%$ as the maximum variation on the number of unprotected coital events.
We summarize the results in the following. First, our estimates are comparable to those in the literature. Based on the data collected from the same project, Tu et al. [38] estimated the per-encounter acquisition risk to be 0.009 , which is between the estimates for 19 years old and 20 years old in our study. We think this is reasonable, considering the average enrollment age is 15 and average stay duration is 3.2 years. Kretzschmar et al. [15] estimated the upper bound for the per-contact probability of transmission to be 0.108 from male to female via casual sex contacts without condom use. Considering an approximately $7.6 \%$ of chlamydia prevalence in the age group of 15-39 cited by the authors in [15], we reasoned that the prevalence in the age group of $15-25$ would be higher, approaching $10 \%$. Then an upper bound on the acquisition risk based on their estimate would have been approximately 0.01 . See Additional file 1 for a list of transmissibility estimates in the literature. Second, our results support the well-established evidence that age is an inverse independent predictor of chlamydial infection [51]. One of the most robust epidemiologic characteristics of chlamydial infection is higher prevalence among younger persons than older ones [50]. The inverse relationship between age and prevalence suggests that protective immunity is acquired over time. Third, our results support the hypothesis that protective immunity is partial at best [53]. Additional analysis with our data set also supported the evidence that repeated infections were strongly related with resumption of sexual activity [22], e.g., we found a similar level of sexual activity was resumed shortly after the treatment.

\section{Discussion}

In this paper, we proposed a Markov-based individual state-transition model with age-varying transition probabilities to estimate age-specific risks of per-encounter $C$. trachomatis acquisition. To calibrate the model, we solved an optimization problem to identify the acquisition risks with which the simulated infection incidences match the observed ones at discrete age points. We conducted our case studies based on the data collected in a longitudinal study of recurrent STI among inter-city adolescent women. We further extended the model to study the differentiation between first-time infection and repeated infection.

There are a few issues that could potentially limit the use of the proposed method in practice. First, if an estimate

Table 1 Estimated risk of per-encounter C. trachomatis acquisition $(\bar{p})$ (1st Model)

\begin{tabular}{|c|c|c|c|c|c|c|c|c|c|c|c|}
\hline \multicolumn{2}{|c|}{ Age (yrs) } & \multirow{2}{*}{$\begin{array}{l}15 \\
0.0585\end{array}$} & \multirow{2}{*}{$\begin{array}{l}16 \\
0.0352\end{array}$} & \multirow{2}{*}{$\begin{array}{l}17 \\
0.0243\end{array}$} & \multirow{2}{*}{$\begin{array}{l}18 \\
0.0168\end{array}$} & \multirow{2}{*}{$\begin{array}{l}19 \\
0.0110\end{array}$} & \multirow{2}{*}{$\begin{array}{l}20 \\
0.0074\end{array}$} & \multirow{2}{*}{$\begin{array}{l}\mathbf{2 1} \\
0.0052\end{array}$} & \multirow{2}{*}{$\begin{array}{l}22 \\
0.0039\end{array}$} & \multirow{2}{*}{$\begin{array}{l}23 \\
0.0024\end{array}$} & \multirow{2}{*}{$\begin{array}{l}\mathbf{2 4} \\
0.0016\end{array}$} \\
\hline$\overline{\bar{p}}$ & $q=0 \%$ & & & & & & & & & & \\
\hline & $q=5 \%$ & 0.0578 & 0.0344 & 0.0241 & 0.0167 & 0.0107 & 0.0072 & 0.0051 & 0.0034 & 0.0023 & 0.0015 \\
\hline & $q=10 \%$ & 0.0569 & 0.0342 & 0.0239 & 0.0164 & 0.0106 & 0.0071 & 0.0050 & 0.0032 & 0.0022 & 0.0014 \\
\hline
\end{tabular}


Table 2 Estimated per-encounter acquisition risks of C. trachomatis causing first-time infection $\left(\bar{p}^{f}\right)$ and repeated infection $\left(\bar{p}^{r}\right)$ (2nd Model)

\begin{tabular}{|c|c|c|c|c|c|c|c|c|c|c|c|}
\hline \multicolumn{2}{|c|}{ Age (yrs) } & \multirow{2}{*}{$\begin{array}{l}17 \\
0.0266\end{array}$} & \multirow{2}{*}{$\begin{array}{l}17.5 \\
0.0216\end{array}$} & \multirow{2}{*}{$\begin{array}{l}18 \\
0.0193\end{array}$} & \multirow{2}{*}{$\begin{array}{l}\mathbf{1 8 . 5} \\
0.0169\end{array}$} & \multirow{2}{*}{$\begin{array}{l}19 \\
0.0142\end{array}$} & \multirow{2}{*}{$\begin{array}{l}19.5 \\
0.0122\end{array}$} & \multirow{2}{*}{$\begin{array}{l}20 \\
0.0100\end{array}$} & \multirow{2}{*}{$\begin{array}{l}\mathbf{2 0 . 5} \\
0.0075\end{array}$} & \multirow{2}{*}{$\frac{21}{0.0059}$} & \multirow{2}{*}{$\begin{array}{l}\mathbf{2 1 . 5} \\
0.0049\end{array}$} \\
\hline$\overline{\bar{p}^{f}}$ & & & & & & & & & & & \\
\hline \multirow[t]{3}{*}{$\bar{p}^{r}$} & $q=0 \%$ & 0.0211 & 0.0133 & 0.0093 & 0.0070 & 0.0049 & 0.0038 & 0.0032 & 0.0028 & 0.0023 & 0.0018 \\
\hline & $q=5 \%$ & 0.0185 & 0.0120 & 0.0086 & 0.0063 & 0.0045 & 0.0035 & 0.0029 & 0.0025 & 0.0021 & 0.0016 \\
\hline & $q=10 \%$ & 0.0159 & 0.0102 & 0.0072 & 0.0055 & 0.0126 & 0.0031 & 0.0024 & 0.0021 & 00018 & 0.0044 \\
\hline
\end{tabular}

on C. trachomatis transmissibility is requested, one must acquire knowledge on the prevalence of $C$. trachomatis among the male partner population for each age-dependent female population subgroup, which is essential to the conversion of age-specific acquisition risk into the agespecific transmission probability. Even in latest observational studies, it remains challenging to acquire accurate assessment on the $C$. trachomatis prevalence for the entire male partner population especially for the female cohort with extended casual sexual relationships. However, the proposed method provides a framework for "what-if" scenario analysis. Given hypothesized prevalence data, one can easily calculate the age-specific transmission probability and further conduct economic analysis on preventive programs/strategies. Second, our estimates are clearly affected by the accuracy of STI testing and behavioral reporting. It is worth noting that the researchers conducting the YWP undertook rigorous laboratory procedures to ensure the former accuracy. Meanwhile, Tu et al. [38] did not find much reporting bias among the study subjects when comparing self-reported coital counts attained from interviews at quarterly visits with those from subjects' daily diaries in the same period. From a modeling point of view, inaccuracy of STI testing can be incorporated in the Markov state-transition model with updated transition probabilities for $\operatorname{Pr}\left(s_{i+1}=C \mid s_{i}=C\right)=\left(1-p_{i}\right)^{n_{i}}+$ $\left(1-\left(1-p_{i}\right)^{n_{i}}\right) \times q^{\prime}$ where $q^{\prime}$ is the false negative rate of the testing. As for modeling of behavior misreporting, since it is about the issue of mainly underreporting, a multiplier between 0 and 1 can be added to $n(t)$ for the adjustment. Finally, we did not have data on the infection status of the sex partners of the study subjects. As a result, we were unable to build a finer-grained stochastic network type model that incorporates various aspects of inter-subject variations, e.g., we assumed a constant probability of having an infected partner for all sexual relationships. Ghani et al. [41] and Turner et al. [17] provide decent references on realistic sexual network models. Furthermore, it should be noted that we did assume a normal distribution on the number of sex acts among the study subjects. However, it was difficult to verify this assumption. Furthermore, we only varied the baseline for the subjects but did not incorporate potential differences on the temporal correlations of sex acts. A boarder class of distributions would be needed to protect the model from misspecification.

Despite of these limitations, we present a flexible and expandable platform for investigating various aspects of bacterial STI transmission. In our future research, we will address the aforementioned limitations with more systematic study of chlamydial infection data and more systematic analysis of realistic stochastic sexual network models.

\section{Additional files}

Additional file 1: C. trachomatis transmissibility in the literature.

Additional file 2: Parameter values of the cubic spline model

$n(t)=\exp \left\{\beta_{0}+\beta_{1} t+\beta_{2} t^{2}+\beta_{3} t^{3}+\sum_{i=1}^{N} u_{i}\left(\left(t-k_{i}\right)_{+}\right)^{3}\right\}$.

\section{Competing interests}

In the past five years, none of the authors have received reimbursements, fees, funding, or salary from an organization that may in some way gain or lose financially from the publication of this manuscript, either now or in the future. None of the authors hold any stocks or shares in an organization that may in some way gain or lose financially from the publication of this manuscript, either now or in the future. None of the authors is currently applying for any patents relating to the content of the manuscript. None of the authors has other financial competing interests.

\section{Authors' contributions}

YT carried out the mathematical model development, simulation input data analysis and model fitting. NK drafted the manuscript and made most of the revisions. WT provided the initial data for analysis and his expert opinion on model development and fitting. All authors read and approved the final manuscript.

\section{Acknowledgements}

The authors thank the referees for their helpful comments that improved the paper. The authors also thank the journal's editorial team for their patience. The data was originally collected through the Young Women Project which was supported by grant RO1 HD042404 from the US National Institutes of Health.

\section{Author details}

'Weldon School of Biomedical Engineering, Purdue University, 206 S. Martin Jischke Dr, West Lafayette, IN 47907, USA. 'Division of Biostatistics, School of Medicine, Indiana University, 340 W 10th Street, Indianapolis, IN 46202, USA.

Received: 15 September 2012 Accepted: 7 April 2014

Published: 25 April 2014 


\section{References}

1. World Health Organization: Global Prevalence and Incidence of Selected Curable Sexually Transmitted Infections: Overview and Estimates. Geneva: World Health Organization; 2001.

2. Black CM, Marrazzo J, Johnson RE, Hook EW 3rd, Jones RB, Green TA, Schachter J, Stamm WE, Bolan G, St Louis ME, Martin DH: Head-to-head multicenter comparison of DNA probe and nucleric acid amplification tests for Chlamydia trachomatis infection in women performed with an improved reference standard. J Clin Microbiol 2002, 40(10):3757-3763.

3. Watson EJ, Templeton A, Russell I, Paavonen J, Mardh PA, Stary A, Pederson BS: The accuracy and efficacy of screening tests for Chlamydia trachomatis: a systemmatic review. J Med Microbiol 2002, 51(12):1021-1031.

4. Wiesenfeld HC, Lowry DL, Heine RP, Krohn MA, Bittener H, Kelligner K, Shultz M, Sweet RL: Self-collection of vaginal swabs for the detection of Chlamydia, gonorrhea, and trichomoniasis: opportunity to encourage sexually transmitted disease testing among adolescents. Sex Transm Dis 2001, 28(6):321-325.

5. Shafer MA, Tebb KP, Pantell RH, Wibbelsman CJ, Neuhaus JM, Tipton AC, Kunin SB, Ko TH, Schweppe DM, Bergman DA: Effect of a clinical practice improvement intervention on Chlamydial screening among adolescent girls. JAMA 2002, 288(22):2846-2852.

6. Centers for Disease Prevention and Control: Trends in Reportable Sexually Transmitted Diseases in the United States, 2006. Altanta: US Dept of Health and Human Services, Centers for Disease Prevention and Control; 2007.

7. Cates W Jr, Wasserheit JN: Genital chlamydial infections: epidemiology and reproductive sequelae. Am J Obstet Gynecol 1991, 164(6, Pt 2):1771-1781.

8. Weinstock H, Berman S, Cates W Jr: Sexually transmitted diseases among American youth: incidence and prevalence estimates, 2000. Perspect Sex Reprod Health 2004, 36(1):6-10.

9. Hillis SD, Owens LM, Marchbanks PA, Amsterdam LF, MacKenzie WR: Recurrent chlamydial infections increase the risks of hospitalization for ectopic pregency and pelvic inflammatory disease. Am J Obstet Gynecol 1997, 176(1, Pt 1):103-107.

10. Paavonen J, Westrom L, Eschenbach D: Pelvic inflammatory disease. In Sexually Transmitted Diseases. 4th edition. Edited by Holmes KK, Sparling PF, Stamm WE, Piot P, Wasserheit JN, Corey L, Cohen M, Watts H. New York, NY: McGraw-Hill; 2008:1017-1050.

11. Fleming DT, Wasserheit JN: From epidemiological synergy to public health policy and practice: the contribution of other sexually transmitted diseases to sexual transmission of HIV infection. Sex Transm Infect 1999, 75(1):3-17.

12. McClelland RS, Sangare L, Hassan WM, Lavreys L, Mandaliya K, Kiarie J, Ndinya-Achola J, Jaoko W, Baeten JM: Infection with Trichomonas vaginalis increases the risk of HIV-1 acquisition. J Infect Dis 2007, 195(5):698-702.

13. Van Der Pol B, Kwok C, Pierre-Louis B, Rinaldi A, Salata RA, Chen PL, van de Wijgert J, Mmiro F, Mugerwa R, Chipato T, Morrison CS: Trichomonas vaginalis infection and human immunodeficiency virus acquisition in African women. J Infect Dis 2008, 197(4):548-554.

14. Kretzschmar M, Welte R, van den Hoek A, Postma MJ: Comparative Model-based Analysis of Screening Programs for Chlamydia trachomatis Infections. Am J Epidemiol 2001, 153(1):90-101.

15. Kretzschmar M, van Duynhoven YT, Severijnen AJ: Modeling prevention strategies for gonorrhea and Chlamydia using stochastic network simulations. Am J Epidemiol 1996, 144(3):306-317.

16. Low N, McCarthy A, Macleod J, Salisbury C, Campbell R, Roberts TE, Horner P, Skidmore S, Sterne JAC, Sanford E, Ibrahim F, Holloway A, Patel R, Barton PM, Robinson SM, Mills N, Graham A, Herring A, Caul EO, Davey Smith G, Hobbs FD, Ross JD, Egger M: Epidemiological, social, diagnostic and economic evaluation of population screening for genital chlamydial infection. Health Technol Assess 2007, 11(8):1-165. iii-iv, ix-xii.

17. Turner KM, Adams EJ, Gay N, Ghani AC, Mercer C, Edmunds WJ: Developing a realistic sexual network model of chlamydia transmission in Britain. Theory Biol Med Model 2006, 3:3.

18. Hu D, Hook EW 3rd, Goldie SJ: Screening for Chlamydia trachomatis in women 15 to 29 years of age: A cost-effectiveness analysis. Ann Intern Med 2004, 141(7):501-513.

19. Hu D, Hook EW 3rd, Goldie SJ: The impact of natual history parameters on the cost-effectiveness of Chlamydia trachomatis screening strategies. Sex Transm Dis 2006, 33(7):428-436.

20. Centers for Disease Control and Prevention: Chlamydia Prevalence Monitoring Project Annual Report: Sexually Transmitted Disease Surveillance Report Supplement. Atlanta: Centers for Disease Control and Prevention; 2001.
21. Hillis SD, Nakashima A, Marchbanks PA, Addiss DG, Davis JP: Risk factors for recurrent Chlamydia trachomatis infections in women. Am J Obstet Gynecol 1994, 170(3):801-806

22. Whittington WL, Kent $C$, Kissinger $P$, Oh MK, Fortenberry JD, Hillis SE, Litchfield B, Bolan GA, St Louis ME, Farley TA, Handsfield HH: Determinants of persistent and recurrent Chlamydia trachomatis infection in young women: results of a multicenter cohort study. Sex Transm Dis 2001, 28(2):117-123.

23. Richey CM, Macaluso M, Hook EW: Determinants of reinfection with Chlamydia trachomatis. Sex Transm Dis 1999, 26(1):4-11.

24. Rietmeijer CA, Van Bemmelen R, Judson FN, Douglas JM Jr: Incidence and repeat infection rates of Chlamydia trachomatis among male and female patients in an STD clinic: implications for screening and rescreening. Sex Transm Dis 2002, 29(2):65-72.

25. Burstein GR, Gaydos CA, Diener-West M, Howell MR, Zenilman JM, Quinn TC: Incident Chlamydia trachomatis infections among inner-city adolescent females. JAMA 1998, 280(6):521-526.

26. Burstein GR, Zenilman JM, Gaydos CA, Diener-West M, Howell MR, Brathwaite W, Quinn TC: Predictors of repeat Chlamydia trachomatis infections diagnosed by DNA amplification testing among inner city females. Sex Transm Infect 2001, 77(1):26-32.

27. Niccolai LM, Hochberg AL, Ethier KA, Lewis JB, Ickovics JR: Burden of recurrent Chlamydia trachomatis infections in young women: further uncovering the "hidden epidemic". Arch Pediatr Adolesc Med 2007, 161(3):246-251.

28. Welte R, Kretzschmar M, Leidl R, van den Hoek A, Jager JC, Postma MJ: Cost-effectiveness of screening programs for Chlamydia trachomatis: a population-based dynamic approach. Sex Transm Dis 2000, 27(9):518-529.

29. Howell MR, Quinn TC, Gaydos CA: Screening for Chlamydia trachomatis in asymptomatic women attending family planning clinics: a cost-effectiveness analysis of three strategies. Ann Intern Med 1998, 128(4):277-284.

30. Buhaug H, Skjeldestad FE, Halvorsen LE, Dalen A: Should asymptomatic patients be tested for Chlamydia trachomatis in general practice? Br J Gen Pract 1990, 40(333):142-145.

31. Buhaug H, Skjeldestad FE, Backe B, Dalen A: Cost effectiveness of testing for chlamydial infections in asymptomatic women. Med Care 1989, 27(8):833-841

32. Centers for Disease Control and Prevention, Workowski KA: Sexually transmitted diseases treatment guidelines, 2010. MMWR Morb Mortal Wkly Rep 2010, 59(RR-12):1-116.

33. US Preventive Services Task Force: Screeing for chlamydial infection: US Preventive Services Task Force Recommndaiton Statement. Ann Intern Med 2007, 147(2):128-134.

34. Meyers D, Wolff T, Gregory K, Marion L, Moyer V, Nelson H, Petitti D, Sawaya GF: USPSTF recommendations for STI screening. Am Fam Physician 2008, 77(6):819-824.

35. Elster AB, Kuznets NJ: AMA Guidelines for Adolescent Preventive Services (GAPS): Recommendations and Rationale. Baltimore, MD: Williams \& Wilkins; 1994.

36. Green M, Palfrey JS, Bright Futures: Guidelines for Health Supervision of Infants, Children, and Adolescents. 2nd edition. National Center for Education in Maternal and Child Health: Arlington, VA; 2000.

37. Katz BP: Estimating transmission probabilities for chlamydial infection. Stat Med 1992, 11(5):567-577.

38. Tu W, Ghosh P, Katz BP: A stochastic model for assessing Chlamydia trachomatis transmission risk by using longitudinal observational data. J R Stat Soc Ser A Soc Stat 2010, 174(4):975-989.

39. van Zessen G, Sandfort TGM: Seksualiteit in Nederland: Seksuseel gedrag, risico en preventie van AIDS. (In Dutch). Amsterdam, The Netherlands: Swets \& Zeitlinger; 1991.

40. Quinn TC, Gaydos C, Shepherd M, Bobo L, Hook EW 3rd, Viscidi R, Rompalo A: Epidemiologic and microbiologic correlates of Chlamydia trachomatis infection in sexual partnerships. JAMA 1996, 276(21):1737-1742.

41. Ghani AC, Swinton J, Garnett GP: The role of sexual partnership networks in the epidemiology of gonorrhea. Sex Transm Dis 1997, 24(1):45-56.

42. Adams EJ, Charlett A, Edmunds WJ, Hughes G: Chlamydia trachomatis in the United Kingdom: a systematic review and analysis of prevalence studies. Sex Transm Infect 2004, 80:354-362.

43. Johnson AM, Mercer CH, Erens B, Copas AJ, McManus S, Wellings K, Fenton KA, Korovessis C, Macdowall W, Nanchahal K, Purdon S, Field J: Sexual behaviour in Britain: partnerships, practices, and HIV risk behaviours. Lancet 2001, 358 (9296):1835-1842. 
44. Gray RT, Beagley KW, Timms P, Wilson DP: Modeling the impact of potential vaccines on epidemics of sexually transmitted Chlamydia trachomatis infection. J Infect Dis 2009, 199(11):1680-1688.

45. Lycke $E$, Löwhagen $G B$, Hallhagen $G$, Johannisson G, Ramstedt K: The risk of transmission of genital Chlamydia trachomatis infection is less than that of genital Neisseria gonorrhoeae infection. Sex Transm Dis 1980, 7(1):6-10.

46. Ruijs GJ, Schut IK, Schirm J, Schröder FP: Prevalence, incidence, and risk of acquiring urogential gonococcal or chlamydial infection in prostitutes working in brothels. Genitourin Med 1988, 64(1):49-51.

47. Vickerman P, Ndowa F, O'Farrell N, Steen R, Alay M, Delany-Moretlew S: Using mathematical modelling to estimate the impact of periodic presumptive treatment on the transmission of sexually transmitted infections and HIV among female sex workers. Sex Transm Infect 2010, 86(3):163-168.

48. Padian NS, Shiboski SC, Jewell NP: The effect of number of exposures on the risk of heterosexual HIV transmission. J Infect Dis 1990, 166(5):883-887.

49. Padian NS, Shiboski SD, Jewell NP: Female-to-male transmission of human immunodeficiency virus. JAMA 1991, 266(12):1664-1667.

50. Lau CY, Qureshi AK: Azithromycin versus doxycycline for genital chlamydial infection. Sex Transm Dis 2002, 29(9):497-502.

51. Datta SD, Sternberg M, Johnson RE, Berman S, Papp JR, McQuillan G, Weinstock H: Gonorrhea and chlamydia in the United States among persons 14 to 38 years of age, 1999 to 2002. Ann Intern Med 2007, 147(2):89-96.

52. Arno JN, Katz BP, McBride R, Carty GA, Batteiger BE, Caine VA, Jones RB: Age and clinical immunity to infections with Chlamydia trachomatis. Sex Transm Dis 1994, 21(1):47-52.

53. Katz BP, Batteiger $B E$, Jones RB: Effect of prior sexually transmitted disease on the isolation of Chlamydia trachomatis. Sex Transm Dis 1987, 14(3):160-164

54. Batteiger $B E, X u$ F, Johnson RE, Rekart ML: Protective immunity to Chlamydia trachomatis genital infection: Evidenc from human studies. $J$ Infect Dis 2010, 201(S2):S178-S189.

55. Barnes RC, Katz BP, Rolfs RT, Batteiger BE, Caine VA, Jones RB: Quantitative culture of endocervical Chlamydia trachomatis. J Clin Microbiol 1990, 28(4):774-780.

doi:10.1186/2043-9113-4-7

Cite this article as: Teng et al: Estimating age-dependent per-encounter chlamydia trachomatis acquisition risk via a Markov-based state-transition model. Journal of Clinical Bioinformatics 2014 4:7.

\section{Submit your next manuscript to BioMed Central and take full advantage of:}

- Convenient online submission

- Thorough peer review

- No space constraints or color figure charges

- Immediate publication on acceptance

- Inclusion in PubMed, CAS, Scopus and Google Scholar

- Research which is freely available for redistribution 